\title{
Étude de l'action des ganglions cérébroïdes et des corps dorsaux sur la synthèse d'ADN induite par la chaleur dans l'ovotestis d'Helix aspersa en hibernation
}

\author{
P Gomot, B Griffond, C Colard, L Gomot
}

\begin{abstract}
SDI CNRS 6319, faculté des sciences, laboratoire de zoologie et embryologie, place Maréchal Leclerc, 25030 Besançon Cedex, France
\end{abstract}

(Reçu le 8 juillet 1991; accepté le 24 décembre 1991)

\begin{abstract}
Résumé - Chez des escargots en début d'hibernation, l'extirpation totale du cerveau (ganglions cérébroïdes : $G C$ et corps dorsaux associés : $C D$ ) augmente la synthèse d'ADN des spermatogonies lorsque les animaux sont placés à $25^{\circ} \mathrm{C}$ pendant un mois. Cet effet n'est pas observé si les animaux sont maintenus à $5^{\circ} \mathrm{C}$. La réimplantation immédiate de la totalité du cerveau à des escargots cérébrotomisés et placés à $25^{\circ} \mathrm{C}$ ne corrige pas complètement les effets de l'ablation de leur cerveau. L'implantation soit de $C D$ soit de $G C$ à des escargots cérébrotomisés montre que les $C D$ ramènent les synthèses d'ADN et les muliplications spermatogoniales au niveau des témoins, tandis que les GC les stimulent. Les GC et les $C D$ exercent donc des effets antagonistes qui contrôlent la synthèse d'ADN dans les spermatogonies de l'escargot durant l'hibernation.
\end{abstract}

Helix aspersa / spermatogenèse / cerveau / hibernation / température

Summary - Effect of the cerebral ganglia and dorsal bodies on temperature-induced DNA synthesis in the gonads of hibernating Helix aspersa. Brain extirpation of snails at the start of their natural hibernation increased the synthesis of DNA in spermatogonia when the animals were transferred from 5 to $25^{\circ} \mathrm{C}$ for a 4-week period. This effect did not occur if animals were maintained at $5{ }^{\circ} \mathrm{C}$. The reimplantation of brain (cerebral ganglia: $C G+$ associated dorsal bodies: $D B$ ) in brainablated snails failed to correct the effects of brain extirpation. The implantation of either DB or CG in cerebrotomized hosts showed that, compared to shams, DB restored the level of DNA synthesis and spermatogonial proliferations whereas CG stimulated it. The CG and associated DB were therefore found to exert antagonistic effects which are responsible for the control of spermatogonial DNA synthesis in hibernating Helix aspersa.

Helix aspersa / spermatogenesis / brain / hibernation / temperature 


\section{INTRODUCTION}

Chez les Mollusques pulmonés, la reproduction et la gamétogenèse dépendent des facteurs de l'environnement (Joosse, 1984; Gomot et al, 1989) qui agissent par l'intermédiaire du système endocrine dont on connaît plusieurs centres producteurs d'hormones (Joosse, 1988).

Chez Helix aspersa en dormance, nous avons observé que la méiose mâle est inhibée par les basses températures ( 6 à $10^{\circ} \mathrm{C}$ ), tandis que les températures supérieures à $16^{\circ} \mathrm{C}$ stimulent la multiplication des spermatogonies et la spermiogenèse (Gomot et al, 1986). Après extirpation du cerveau, nous avons ensuite montré, par mesure d'incorporation de thymidine tritiée et observation autoradiographique, que celui-ci exerce une action inhibitrice sur la multiplication des spermatogonies et des spermatocytes induite par la chaleur au début de l'hibernation (Gomot et Gomot, 1989).

L'objet du présent travail est de chercher comment interviennent les 2 constituants principaux du système neuroendocrine cérébral que sont les ganglions cérébroïdes (GC) et les corps dorsaux (CD) de la gaine conjonctive périganglionnaire. Pour cela, nous avons procédé d'une part à l'extirpation du cerveau et d'autre part à sa réimplantation ou à celle des GC ou des CD. Nous avons vérifié l'état de survie et le fonctionnement des tissus transplantés par des études ultrastructurales et cyto-immunologiques. Pour ces dernières, nous avons choisi de rechercher deux peptides, le FMRFamide et la méthionine-enképhaline, dont nous connaissons la localisation dans le cerveau des escargots et qui jouent un rôle important dans le contrôle des corrélations endocrines (Marchand et Dubois, 1986; Griffond et Mounzih, 1989; Marchand et al, 1991). Nous avons ensuite comparé les effets des différentes interventions sur la multiplication des spermatogonies.

\section{MATÉRIEL ET MÉTHODES}

\section{Les animaux}

Les expériences ont été réalisées sur des escargots Helix aspersa nés au printemps, élevés en parcs extérieurs et récoltés à l'état adulte à leur entrée en hibernation naturelle au mois d'octobre. Ils ont été ensuite conservés un mois en chambre froide à $5^{\circ} \mathrm{C}$ à l'obscurité avant la réalisation des expériences.

\section{Conditions expérimentales}

Les escargots en hibernation sont réveillés pendant quelques heures afin de pratiquer les interventions sur le cerveau selon la technique décrite précédemment (Gomot ef al, 1990a). Aussitôt après extirpation ( $E$ ) du cerveau, des escargots reçoivent l'implantation dans l'hémocoele d'un cerveau entier (groupe $\mathrm{E}+\mathrm{I}, n=12$ ), tandis qu'à d'autres on implante seulement les ganglions cérébroïdes (groupe $E+G C, n=12$ ) ou le conjonctif périganglionnaire cérébral contenant les corps dorsaux (groupe $\mathrm{E}+\mathrm{CD}, n=12$ ). Des animaux témoins ( $T, n=12$ ) subissent uniquement l'anesthésie et la section du tégument. Après l'opération, les escargots sont conservés à $5{ }^{\circ} \mathrm{C}$ pendant une semaine. Ensuite, 2 lots d'animaux témoins et opérés sont constitués; l'un demeure à $5^{\circ} \mathrm{C}(n=24)$, tandis que l'autre est placé à $25^{\circ} \mathrm{C}(n=24)$.

Après 4 semaines à $5^{\circ} \mathrm{C}$ ou $25^{\circ} \mathrm{C}, 6$ escargots de chaque groupe expérimental reçoivent dans l'hémocoele de la région viscérale une injection de thymidine-3H du CEA (Commissariat à l'Énergie Atomique - 91191 Gif-sur-Yvette) d'activité spécifique $1 \mathrm{Ci} / \mathrm{mmol}$, à raison de 5 $\mu \mathrm{Ci} / \mathrm{g}$ de poids frais dans $0,02 \mathrm{ml}$ de liquide de Ringer. Cinq heures plus tard, au moment du sacrifice, on prélève 2 morceaux des organes étudiés : un est fixé pour les examens cytologique et radioautographique, l'autre est pesé pour la détermination de la teneur en ADN et pour la mesure de la thymidine- ${ }^{3} \mathrm{H}$ incorporée. 
Étude comparée de la synthèse d'ADN dans la gonade et la glande à albumen (organe témoin) par mesure de l'incorporation de thymidine- ${ }^{3} \mathrm{H}$

L'évaluation de la synthèse d'ADN est faite par la mesure de l'incorporation de thymidine- ${ }^{3} \mathrm{H}$ (Sokolove et al, 1983). Ces valeurs d'incorporation sont rapportées à la quantité d'ADN dosée par spectrofluorimétrie (Brunk et al, 1979). Les comparaisons des résultats, exprimés en dpm/pg d'ADN, sont faites par application du test non paramétrique $U$ de Mann et Withney (Siegel, 1956).

\section{Étude cytologique et radioautographique}

Les organes subissent une double fixation glutaraldéhyde-acide osmique avant d'être inclus dans l'ERL 4206 et traités pour les examens cytologiques et radioautographiques selon les procédés déjà décrits (Gomot et al, 1990b). La comparaison du nombre et de la surface des organites des cellules des corps dorsaux est réalisée par analyse d'images sur photos de microscopie électronique et application du test $(t)$ de Student.

\section{Observations cyto-immunologiques}

Des cerveaux témoins et des greffons sont fixés dans du liquide de Bouin-Hollande/sublimé puis débités en coupes de $7 \mu \mathrm{m}$ d'épaisseur. Les coupes sont traitées en immuno-enzymologie selon la procédure décrite par Marchand et al (1982) pour le FMRFamide et Marchand et Dubois (1986) pour la méthionine-enképhaline.

\section{RÉSULTATS}

\section{État des animaux et des greffons}

\section{Survie}

Les escargots cérébrotomisés ou implantés, maintenus en inactivité à 5 ou $25^{\circ} \mathrm{C}$, survivent aussi bien que les témoins : aucune mortalité n'est enregistrée pendant la durée de l'expérience.

\section{Aspect cytologique des transplants cérébraux}

Les organes transplantés ne sont pas rejetés, ils sont retrouvés dans l'hémocoele au moment du sacrifice de l'animal.

L'étude comparée des $C D$ et des GC a été conduite dans les transplants des escargots placés à $25^{\circ} \mathrm{C}$ chez qui l'implantation entraîne des modifications de la synthèse d'ADN de la lignée mâle.

Les cerveaux implantés entiers prennent une forme globuleuse et sont entourés par une épaisse capsule conjonctive. À l'intérieur, les ganglions cérébroïdes limités par une lame basale conservent leur structure et leurs relations avec les cellules des corps dorsaux par l'intermédiaire d'axones. Certains neurones ont une réaction metenképhaline positive, tandis que d'autres sont FMRFamide positifs. Les cellules des corps dorsaux ont le même aspect que dans les cerveaux témoins et présentent une réaction positive à la met-enképhaline. Les organites cytoplasmiques (appareil de Golgi, mitochondries, inclusions lipidiques, grains de sécrétion) y ont le même développement que dans les cerveaux témoins (tableau 1).

Lorsque les ganglions cérébroïdes isolés sont implantés, les neurones survivent bien (fig 1), mais leur disposition est fortement modifiée. L'activité des cellules neurosécrétrices est attestée par le développement important de l'appareil de Golgi et les nombreux grains de sécrétion (fig 2) qui sont libérés par exocytose (fig 3 ) dans leur environnement immédiat.

Les cellules des corps dorsaux (séparés des ganglions cérébroïdes) implantés dans l'hémocoele (fig 4), prennent un aspect 

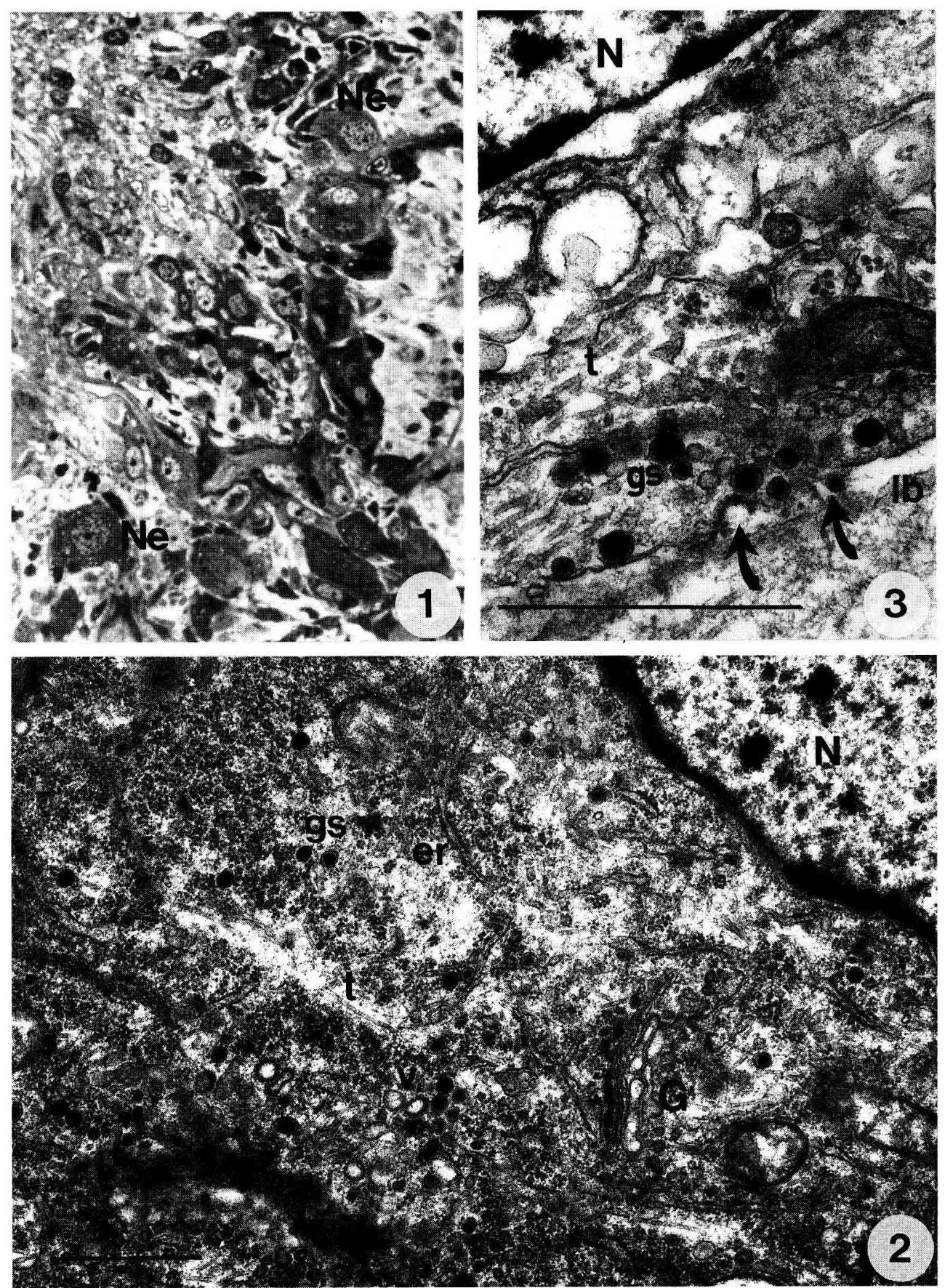
Tableau I. Comparaison du nombre et de la surface d'organites des cellules des corps dorsaux de cerveaux témoins, de cerveaux implantés entiers (CV) et d'implants de corps dorsaux isolés (CDi) après 4 semaines à $25^{\circ} \mathrm{C}$.

\begin{tabular}{|c|c|c|c|c|c|c|}
\hline & \multicolumn{2}{|c|}{ Mitochondries } & \multicolumn{2}{|c|}{ Inclusions lipidiques } & \multirow{2}{*}{$\begin{array}{c}\text { Nombre } \\
\text { de } \\
\text { dictyosomes }\end{array}$} & \multirow{2}{*}{$\begin{array}{c}\text { Nombre } \\
\text { de grains } \\
\text { de sécrétion }\end{array}$} \\
\hline & Nombre & Surface & Nombre & Surface & & \\
\hline $\begin{array}{l}\text { Cerveaux } \\
\text { Témoins }\end{array}$ & $903 \pm 271^{a}$ & $174 \pm 51^{a}$ & $553 \pm 370^{a}$ & $165 \pm 101^{a}$ & $5,5 \pm 12^{a}$ & $762 \pm 327^{a}$ \\
\hline Implants & $884 \pm 88^{a}$ & $120 \pm 45^{a}$ & $389 \pm 93^{a}$ & $216 \pm 109^{a}$ & $13 \pm 14^{a}$ & $1024 \pm 248^{a}$ \\
\hline$\underset{\mathrm{CDi}}{\text { Implants }}$ & $440 \pm 182^{b}$ & $143 \pm 206^{a}$ & $7 \pm \quad 6^{b}$ & $1,56 \pm 1,4^{b}$ & $62 \pm 26^{b}$ & $149 \pm 44^{b}$ \\
\hline
\end{tabular}

Les valeurs ( $m \pm$ sem) sont données pour une surface de coupe de $1000 \mu \mathrm{m}^{2}$; dans une même colonne, la même lettres indique que les moyennes ne sont pas statistiquement différentes, des lettres différentes indiquent que les moyennes diffèrent $(P<0,05)$.

très différent de celui des cellules des CD des cerveaux greffés entiers et des cerveaux témoins. Les changements ultrastructuraux les plus apparents, confirmés par l'étude morphométrique, concernent la forte diminution des enclaves lipidiques (tableau I), le développement de l'appareil de Golgi (fig 5, tableau I), l'apparition de très nombreuses vésicules hérissées et de vésicules de réticulum lisse (fig 5), la diminution du nombre de grains de sécrétion (tableau I). Les mitochondries moins nombreuses ont une surface relative équivalente (tableau I), leur taille augmente et elles changent d'aspect; leur matrice devient dense aux électrons et leurs crêtes s'orientent longitudinalement (fig 6). On re- connaît encore quelques axones en contact étroit avec les cellules des CD, mais ils ne contiennent plus de grains de sécrétion (fig 7).

\section{Évolution des synthèses d'ADN dans l'ovotestis}

\section{Action de la cérébrotomie sur la synthèse d'ADN gonadique en réponse à l'augmentation de la température}

Chez les escargots témoins ou traités maintenus à $5{ }^{\circ} \mathrm{C}$, l'incorporation de thymidine- ${ }^{3} \mathrm{H}$ dans la glande à albumen et dans

Figs 1-3. 1. Lorsque les GC sont implantés dans l'hémocoele, dépourvus de leur gaine conjonctive, ils prennent une forme globuleuse et les neurones ( $\mathrm{Ne}$ ) restent moins étroitement associés les uns aux autres que lorsque le cerveau est implanté en entier. $\times 280.2$. Les cellules neurosécrétrices des GC implantés isolément (fig 1) conservent une activité attestée par le développement des dictyosomes (G) et les nombreux grains de sécrétion (gs). er : ergastoplasme; $N$ : noyau; $t$ : microtubules; $v$ : vésicules. Barre $=1 \mu \mathrm{m}$. 3. Les grains de sécrétion ( $\mathrm{gs}$ ) des cellules neurosécrétrices des $\mathrm{GC}$ implantés sont émis directement par exocytose (flèches) dans les espaces intercellulaires. lb : lame basale; $\mathbf{N}$ : noyau; $\mathbf{t}$ : microtubules. Barre $=1 \mu \mathrm{m}$. 


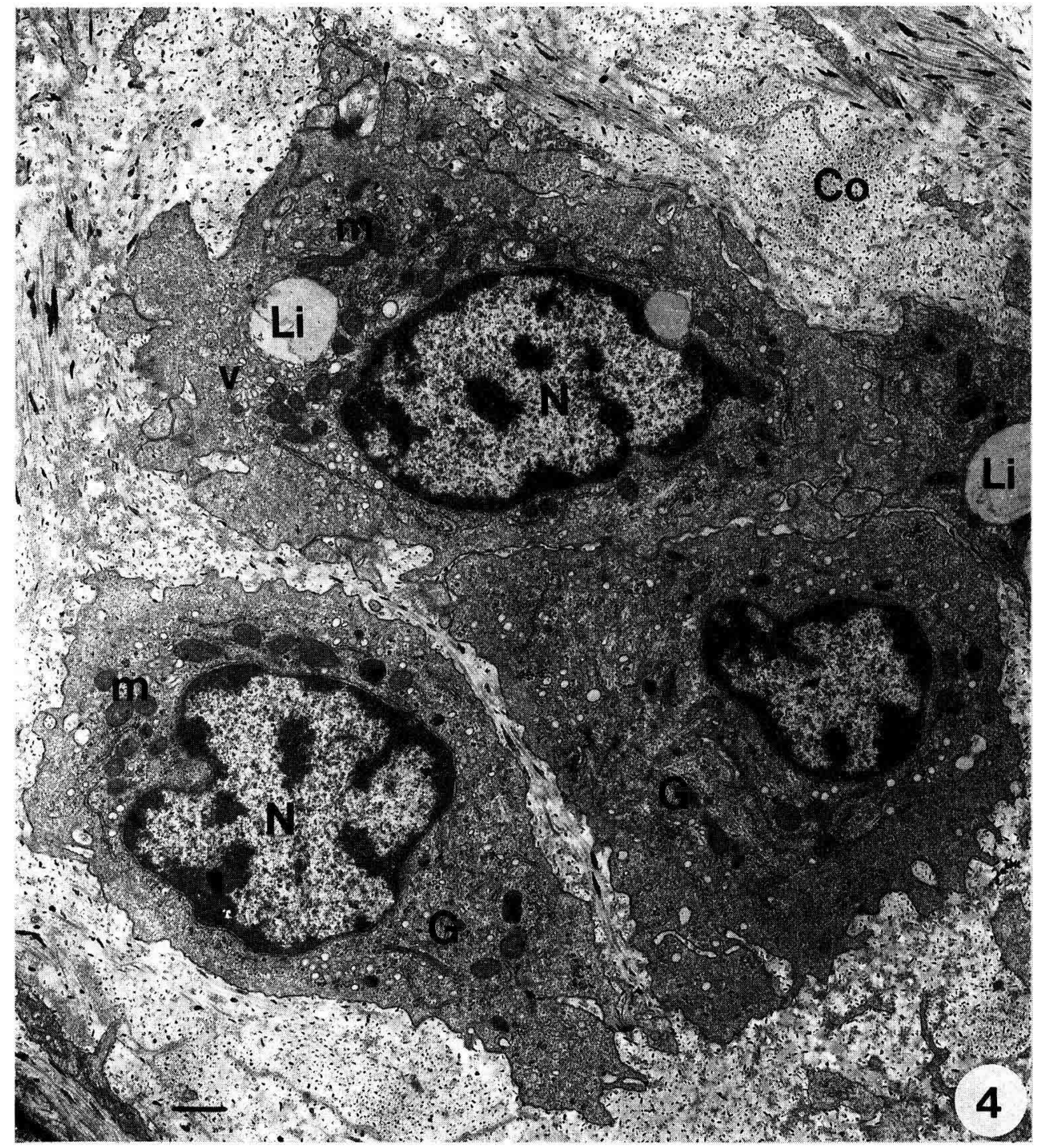

Fig 4. Aspect d'un îlot de 3 cellules de $C D$ entourées de collagène $(C o)$ après implantation pendant 4 semaines à $25^{\circ} \mathrm{C}$ de la gaine périganglionnaire des GC dans l'hémocoele d'un escargot cérébrotomisé. Elles sont très différentes des cellules d'un cerveau implanté entier. Les enclaves lipidiques ont disparu, l'appareil de Golgi (G) est très développé et donne de nombreuses vésicules (v). On n'observe plus d'axones remplis de grains de sécrétion à leur contact. Li : globules lipidiques; $\mathrm{m}$ : mitochondries; $\mathrm{N}$ : noyau. Barre $=1 \mu \mathrm{m}$. 
Tableau II. Comparaison de l'incorporation de thymidine- ${ }^{3} \mathrm{H}$ dans la gonade et la glande à albumen d'escargots placés à $5^{\circ} \mathrm{C}$ et $25^{\circ} \mathrm{C}$ après extirpation du cerveau et réimplantation de ce dernier, entier ou après séparation des corps dorsaux et des ganglions cérébroïdes.

\begin{tabular}{|c|c|c|c|c|c|c|}
\hline \multirow{2}{*}{ Température } & \multirow{2}{*}{ Organe } & \multicolumn{5}{|c|}{$\begin{array}{c}\text { Synthèse d'ADN: dpm/pg ADN } \pm \text { SEM } \\
\text { selon les groupes expérimentaux }{ }^{1}\end{array}$} \\
\hline & & $T$ & $E$ & $E+I$ & $E+G C$ & $E+C D$ \\
\hline \multirow{2}{*}{$\begin{array}{l}5^{\circ} \mathrm{C} \\
4 \text { semaines }\end{array}$} & Gonade & $20 \pm 2^{a}$ & $13 \pm 3^{a}$ & $17 \pm 6^{a}$ & $26 \pm 10^{a}$ & $14 \pm 6^{a}$ \\
\hline & Glande à albumen & $24 \pm 6^{a}$ & $26 \pm 1^{a}$ & $22 \pm 5^{a}$ & $19 \pm$ & $15 \pm 5^{a}$ \\
\hline \multirow{2}{*}{$\begin{array}{l}25^{\circ} \mathrm{C} \\
4 \text { semaines }\end{array}$} & Gonade & $103 \pm 27^{a}$ & $358 \pm 46^{b}$ & $293 \pm 88^{b}$ & $795 \pm 170^{c}$ & $173 \pm 56^{a}$ \\
\hline & Glande à albumen & $13 \pm 5^{a}$ & $17 \pm 5^{a}$ & $14 \pm 5^{a}$ & $27 \pm$ & $25 \pm 3^{a}$ \\
\hline
\end{tabular}

$1 \mathrm{~T}$ : Témoins; $E$ : Extirpation cerveau; $E+1:$ Extirpation + réimplantation cerveau; $E+C G$ : Extirpation cerveau + réimplantation ganglions cérébroïdes; $E+C D$ : Extirpation cerveau + réimplantation corps dorsaux.

Chaque groupe expérimental comporte 6 escargots pour chacune des températures. Dans une même ligne, la même lettre indique que les moyennes ne sont pas statistiquement différentes, des lettres différentes indiquent que les moyennes diffèrent $(P<0,05)$.

la gonade est très faible (tableau II). La spermatogenèse est bloquée au stade spermatocytes.

À $25{ }^{\circ} \mathrm{C}$, chez les témoins, la spermatogenèse s'effectue complètement en 4 semaines (fig 8). Alors que l'incorporation de thymidine $-3 \mathrm{H}$ reste constante dans la glande à albumen, elle est multipliée par 5 dans la gonade (tableau II). Ce sont les cellules mâles, spermatogonies et spermatocytes en début de prophase méiotique, qui incorporent le précurseur radioactif.

La cérébrotomie ne modifie pas la différenciation des spermatozoïdes qui semble comparable à celle des témoins. Par contre, elle provoque une augmentation ( $x$ $3,5)$ de la synthèse d'ADN gonadique qui se traduit par un nombre plus important de spermatogonies et de spermatocytes marqués (fig 9). L'incorporation de thymidine n'est pas affectée dans la glande à albumen.

\section{Action de la réimplantation du cerveau entier, des ganglions cérébroïdes ou des corps dorsaux isolés}

À $5{ }^{\circ} \mathrm{C}$, l'incorporation de thymidine $-3 \mathrm{H}$ dans la gonade et la glande à albumen demeure faible et ne varie pas de façon significative quelle que soit la réimplantation pratiquée (tableau II).

À $25{ }^{\circ} \mathrm{C}$, la réimplantation du cerveau entier dans l'hémocoele d'escargots préalablement cérébrotomisés ne ramène pas le niveau de synthèse d'ADN gonadique à celui des animaux témoins. Elle ne corrige donc que partiellement les effets de l'ablation du cerveau $(E+I$ pas différent de $E$; tableau II).

La réimplantation des ganglions cérébroïdes provoque une stimulation très importante de la synthèse d'ADN dans les cellules mâles : l'incorporation de thymidine- ${ }^{3} \mathrm{H}$ est 2 fois plus importante que chez les 

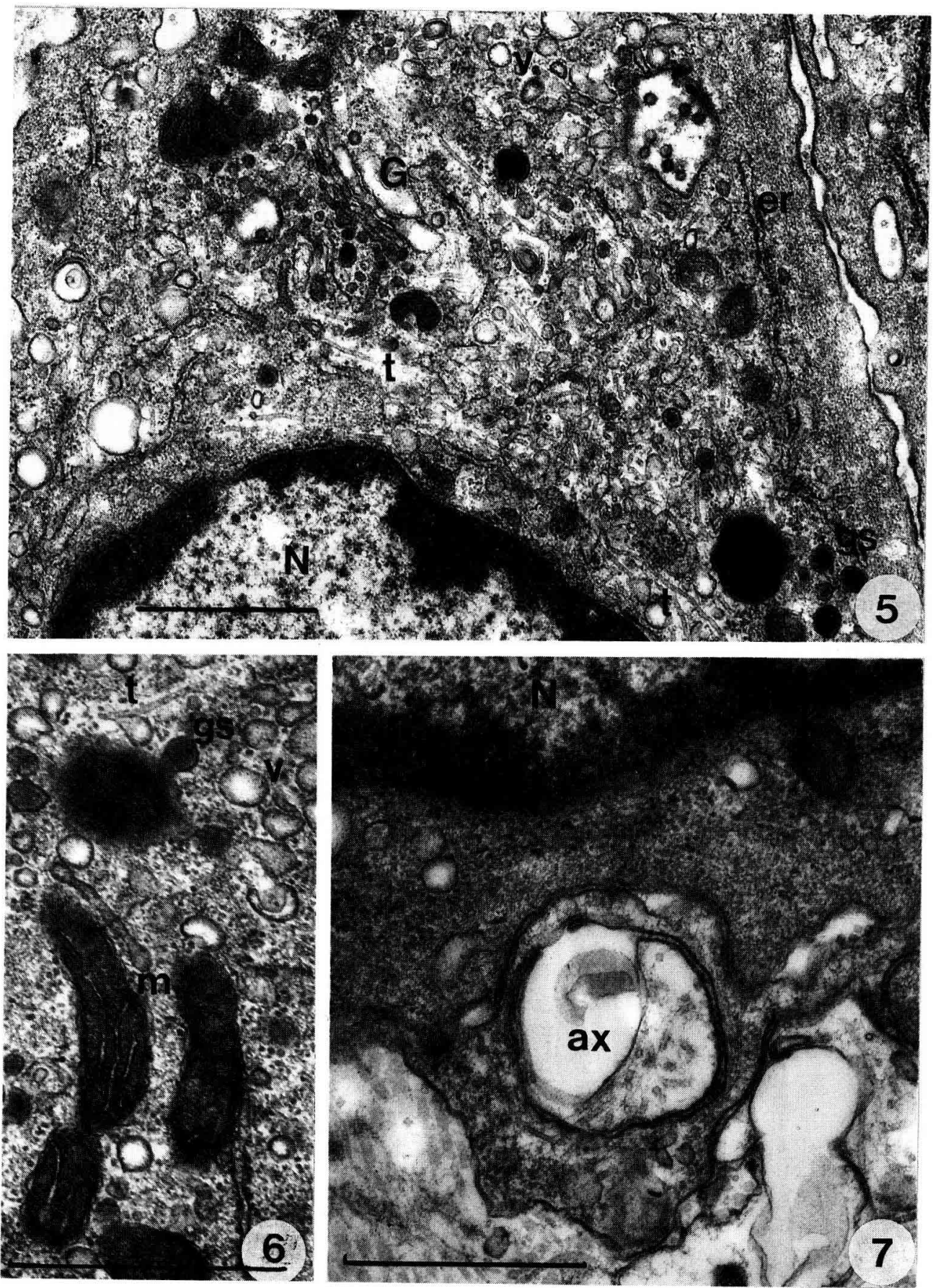
escargots cérébrotomisés et 8 fois plus que chez les témoins (tableau II). Le nombre de spermatogonies et de spermatocytes marqués est élevé (fig 10).

La réimplantation du conjonctif péricérébral contenant les CD diminue significativement la synthèse d'ADN gonadique des escargots cérébrotomisés et la ramène au niveau de celle des animaux témoins (tableau II). Ce même traitement diminue le nombre de cellules mâles marquées (fig 11).

\section{DISCUSSION}

Chez l'escargot (Gomot et al, 1990b), comme chez la limace (Sokolove et al, 1983), lorsque les synthèses d'ADN gonadique sont stimulées expérimentalement, ce sont essentiellement les cellules de la lignée mâle (spermatogonies et spermatocytes en début de prophase méiotique) qui incorporent la thymidine tritiée.

Les expériences d'extirpation du cerveau suivies, soit de la réimplantation du cerveau entier $(G C+C D)$, soit de la réimplantation des $G C$ ou des $C D$, mettent en évidence les fonctions respectives des $\mathrm{GC}$ et des CD dans la régulation de la spermatogenèse des escargots. De plus, elles confirment les résultats antérieurs : rôle de la température dans la réalisation de la spermiogenèse (Gomot et al, 1990b), existence d'une activité cérébrale inhibitrice de la multiplication des spermatogonies et des spermatocytes (Gomot et Gomot, 1989).

L'implantation des GC et des CD isolés apporte des éléments nouveaux qui témoignent de l'existence d'interrelations fonctionnelles étroites entre ces 2 organes. Lorsqu'ils sont séparés, les GC et les CD exercent individuellement une action opposée sur la synthèse d'ADN des spermatogonies et des spermatocytes. Les CD implantés seuls inhibent la synthèse d'ADN. Leurs cellules ont un aspect fortement activé qui peut s'expliquer par la suppression du contrôle inhibiteur de type nerveux exercé par les GC (Vincent et al, 1984b). L'action des GC sur les CD pourrait s'effectuer par l'intermédiaire des neurones FMRFamide positifs et de leurs axones qui sont en contact avec les cellules des CD (Griffond et Mounzih, 1990) car le FMRF amide synthétique inhibe in vitro la synthèse des protéines des CD (Griffond et Mounzih, 1989).

Inversement, l'implantation des GC isolés augmente très nettement la synthèse d'ADN des spermatogonies. Ceci peut résulter de l'absence d'un effet inhibiteur direct des $C D$ sur les cellules mâles et/ou d'un effet stimulateur des GC sur ces mêmes cellules; la seconde hypothèse suggère une influence inhibitrice des $C D$ sur les GC, comme cela avait été supposé par Bohmig (1931) chez Planorbarius. Cette action inhibitrice des CD sur la spermatogenèse opposée à leur action stimulatrice sur la vitellogenèse (Vincent et al,

Figs 5-7. Plus forts grossissements du cytoplasme de cellules des CD après séparation des GC et implantation comme figure 4. er : ergastoplasme; $N$ : noyau; $t$ : microtubules. Barre $=1 \mu \mathrm{m}$. 5 . L'appareil de Golgi $(G)$ forme de nombreux grains de sécrétion (gs) et des vésicules hérissées (v). 6 . Les mitochondries $(\mathrm{m})$ ont une matrice dense et des crêtes qui s'orientent longitudinalement. 7 . On repère encore quelques prolongements axonaux (ax) entourés du cytoplasme des cellules des CD, mais ils ne contiennent plus de grains de sécrétion. 
P Gomot et al
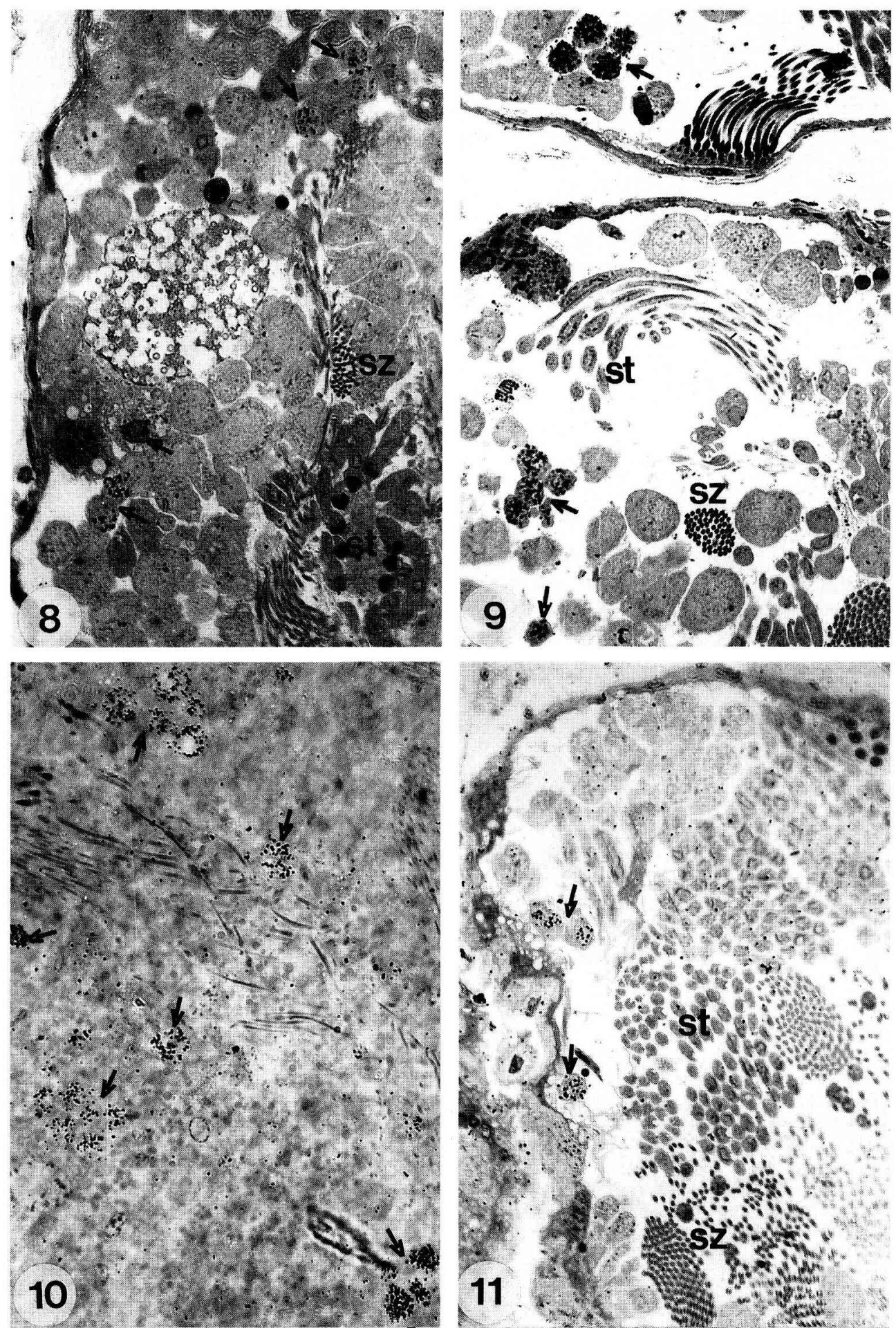
1984a) apporte un élément nouveau de compréhension de la régulation de l'hermaphrodisme chez les escargots.

Les résultats des associations in vitro de CD isolés et de gonades (Vincent et al, 1984a) ou des implantations de GC et de $C D$ séparés décrites dans ce travail mettent en évidence la coexistence dans le cerveau d'Helix aspersa de facteurs stimulateurs et de facteurs inhibiteurs. Aussi, peut-on penser, comme Mathieu (1987) chez Mytilus, que le contrôle de la multiplication goniale fait intervenir 2 facteurs antagonistes dont les taux respectifs déterminent les phases de la gamétogenèse en fonction des conditions d'environnement.

En conclusion, nos expériences apportent la preuve que le cerveau exerce une influence globale inhibitrice sur la multiplication des spermatogonies et des spermatocytes au début de l'hibernation. Cette action semble résulter de 2 influences antagonistes des $\mathrm{CD}$ et des GC qui peuvent produire respectivement un facteur inhibiteur (CD) et un facteur mitogène (GC). Cette action inhibitrice du cerveau contrôle la multiplication des spermatogonies et des spermatocytes pendant la période d'hibernation.

\section{REMERCIEMENTS}

Les auteurs remercient vivement Brigitte Jolibois pour la préparation du manuscrit.

\section{RÉFÉRENCES}

Bohmig L (1931) Zur Kenntnis der sogenannten Dorsallapen des Gehirns von Lymnaea stagnalis (L) Lam und Planorbis corneus (L) Pfeiff. Sber Akad Wiss Wien Math Nat KI 140, 319-335

Brunk CF, Jones KC, James TW (1979) Assay for nano-gram quantities of DNA in cellular homogenates. Anal Biochem 92, 497-500

Gomot $P$, Gomot $L$ (1989) Inhibition of temperature-induced spermatogenic proliferation by a brain factor in hibernating Helix aspersa (Mollusca). Experientia 45, 349-351

Gomot P, Griffond B, Gomot L (1986) Effets de la température sur la spermatogenèse d'Escargots Helix aspersa maintenus en repos artificiel. CR Séances Acad Sci Paris 302, 2732

Gomot P, Gomot L, Griffond B (1989) Evidence for a light compensation of the inhibition of reproduction by low temperatures in the snail Helix aspersa. Ovotestis and albumen gland responsiveness to different conditions of photoperiods and temperatures. Biol Reprod 40, 1237-1245

Figs 8-11. 8. Acinus d'ovotestis d'escargot témoin en hibernation maintenu 4 semaines à $25^{\circ} \mathrm{C}$. Pendant cette période, la méiose s'est effectuée et on note la différenciation de spermatides (st) et de spermatozoïdes (sz). D'autre part, après injection de thymidine- ${ }^{3} \mathrm{H}$ ( 5 heures avant le sacrifice), 6 spermatocytes $(x)$ en phase de synthèse d'ADN sont révélés par le traitement radioautographique. $\times 510$. 9. L'ablation du cerveau, suivie d'un séjour de l'animal pendant 4 semaines à $25^{\circ} \mathrm{C}$, provoque une augmentation du nombre de spermatocytes qui incorporent la thymidine- ${ }^{3} \mathrm{H}(\mathcal{})$ (10 cellules marquées sur la coupe). En l'absence du cerveau, la différenciation des spermatides (st) et des spermatozoïdes (sz) est normale. $\times 510$. 10. Après ablation du cerveau et implantation des GC isolés, après 4 semaines à $25^{\circ} \mathrm{C}$, on observe un grand nombre de spermatocytes (20 sur la zone photographiée) qui ont incorporé de la thymidine- ${ }^{3} \mathrm{H}(\bullet) \times 510$. 11. Lorsque l'ablation du cerveau est accompagnée de l'implantation de CD isolés, on observe peu de spermatocytes $(\pi)$ ayant incorporé de la thymidine-3 $\mathrm{H}$ ( 3 sur la coupe). Les spermatides (st) et les spermatozoïdes (sz) se sont formés normalement. $\times 510$. 
Gomot P, Gomot L, Marchand CR, Colard C (1990a) Extirpation and transplantation of the brain of the snail Helix aspersa: a study of the survival of the animal and the implant. Can J Zool 68, 1505-1512

Gomot P, Griffond B, Gomot L (1990b) Action de la température sur la multiplication des cellutes mâles et la spermatogenèse d'Helix aspersa en hibernation. J Thermal Biol 15, 267-280

Griffond B, Mounzih K (1989) In vitro inhibition of methionine incorporation in the dorsal bodies of Helix aspersa by synthetic FMRF amide. Comp Biochem Physiol 92C, 45-49

Griffond B, Mounzih K (1990) Innervation of the dorsal body cells of Helix aspersa: immunocytochemical evidence for the presence of FMRFamide-like substances in nerves and synapses. Tissue \& Cell 22, 741-748

Joosse J (1984) Photoperiodicity, rhythmicity and endocrinology of reproduction in the snail Lymnaea stagnalis. In: Photoperiodic Regulation of Insect and Molluscan Hormones. Ciba Foundation Symposium, vol 104, Pitman, Londres, 204-220

Joosse J (1988) The hormones of molluscs. In: Endocrinology of selected invertebrate types (H Laufer, GH Downer, eds), Alan R Liss Inc, 89-140

Marchand CR, Dubois MP (1986) Mise en évidence par immunocytochimie de substances apparentées à la méthionine-enképhaline dans divers tissus de l'escargot Helix aspersa Müller. Haliotis 15, 241-247

Marchand CR, Wijdenes J, Schot LPC (1982) Localisation par la technique cyto-immuno- enzymologique d'un neuropeptide cardioexcitateur (le FMRF-amide) dans le collier nerveux périoesophagien d'Helix aspersa Müller (Gastéropode, Pulmoné, Stylommatophore). CR Séances Acad Sci Paris Ser III 294, 39-44

Marchand CR, Griffond B, Mounzih K, Colard C (1991) Distribution of methionine-enkephalinlike and FMRFamide-like immunoreactivities in the central nervous system (including dorsal bodies) of the snail Helix aspersa Müller. Zool Sci 8, 909-913

Mathieu M (1987) Étude expérimentale des contrôles exercés par les ganglions nerveux sur la gamétogenèse et les processus métaboliques associés chez la moule Mytilus edulis. Thèse Doct Etat, Université de Caen

Siegel $S$ (1956) The case of two independent samples in non parametric statistics. International Student Ed, McGraw Hill International Book Co, Auckland, p 116

Sokolove PG, Melrose GR, Gordon TM, O'Neill MC (1983) Stimulation of spermatogonial DNA synthesis in slug gonad by a factor released from cerebral ganglia under the influence of long days. Gen Comp Endocrinol 50, 95-104

Vincent C, Griffond B, Gomot L, Bride J (1984a) Étude in vitro de l'influence des corps dorsaux sur l'ovogenèse d'Helix aspersa Müll. Gen Comp Endocrinol 54, 230-235

Vincent C, Griffond B, Wijdenes J, Gomot L (1984b) Contrôle d'une glande endocrine : les corps dorsaux par le système nerveux central chez Helix aspersa. CR Séances Acad Sci Paris Sér III 299, 421-426 